\title{
Educação Alimentar e Nutricional: influência no comportamento alimentar e no estado nutricional de estudantes
}

\author{
Food and Nutrition Education: influence on students feeding \\ behavior and nutritional status
}

\author{
Maria Irene de Castro Barbosa* \\ Bárbara Ramos de Oliveira** \\ Nágila Araújo de Carvalho** \\ Karine Anusca Martins**
}

\begin{abstract}
Resumo
A transição nutricional indica um consumo alimentar inadequado na população brasileira sendo necessária a adoção de estratégias educativas com a finalidade de favorecer comportamentos saudáveis. O objetivo deste trabalho foi avaliar a influência de um programa de intervenção nutricional educativa no hábito alimentar e indicadores antropométricos de alunos da primeira fase do ensino fundamental. Estudo quantitativo prospectivo, realizado em uma escola federal de Goiânia/Goiás, com 92 alunos. Realizou-se caracterização sociodemográfica, avaliação do peso, altura e Índice de Massa Corporal (IMC) e, para a identificação do hábito alimentar, aplicou-se o Questionário Alimentar do Dia Anterior (QUADA), previamente validado. Um programa de intervenção nutricional educativa foi implantado, sendo

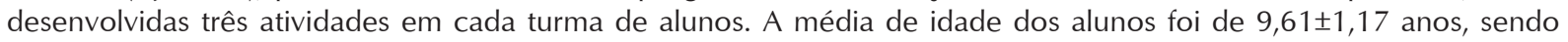
que a maioria eram meninas (55,44\%), da classe social "B" (54,38\%); pardos (57,90\%); católicos $(54,39 \%)$, moravam com pais $(68,42 \%)$ e tinha um a dois irmãos $(71,94 \%)$. A maioria dos participantes apresentou eutrofia, antes e após a intervenção, e 20,00\% apresentou excesso de peso. Após cinco meses da intervenção educativa, observou-se aumento de peso e IMC, sem significância estatística, ganho estatural significativo $(p<0,0001)$. Houve aumento no consumo do grupo de frutas/verduras/hortaliças no almoço e lanche da tarde e diminuição no desjejum e jantar, redução do consumo do grupo do feijão no almoço e aumento no jantar. Houve aumento do consumo de alimentos não saudáveis no desjejum e almoço. Ações de educação alimentar e nutricional devem ser contínuas e inseridas no planejamento pedagógico das escolas para melhores resultados no comportamento alimentar dos escolares.
\end{abstract}

Palavras-chave: Educação Alimentar e Nutricional. Comportamento alimentar. Antropometria.

\begin{abstract}
The nutritional transition indicates inadequate food intake in our population and it requires the adoption of educational strategies in order to promote healthy behaviors. This study evaluated the influence of an educational intervention program in dietary habits and anthropometric indicators in elementary school students. This was a prospective quantitative study developed in a public school in Goiânia, Goiás, with 92 students. Sociodemographic characteristics were analyzed as well as weight, height and body mass index (BMI) measurements. In order to identify the feeding habits, the already validated Previous Day Food Quiz (PDFQ) was used. An educational intervention program was implemented and three activities were developed in each classroom. The students' average age was $9.61 \pm 1.17$ years old, and the majority were girls $(55.44 \%)$, social class "B" $(54.38 \%)$; brown skin $(57.90 \%)$; catholics $(54.39 \%)$, living with their parents $(68.42 \%)$ and they had one to two brothers (71.94\%). Most participants had normal weight before and after the intervention, and $20.00 \%$ were overweight. After five months of the educational intervention, there was an increase in weight and BMI, although not statistically significant, and a significant height increase $(p<0.0001)$. Fruits and vegetables consumed at lunchtime and during the afternoon snack increased, while it decreased at breakfast and dinnertime. The bean group intake at lunchtime decreased and increased at dinnertime. There was an increase in the consumption of unhealthy foods for breakfast and lunch. Food and nutrition education should be continuous and integrated into the pedagogical planning in order to achieve better results in school feeding behavior.
\end{abstract}

Keywords: Food and Nutritional Education. Feeding behavior. Anthropometry.

DOI: $10.15343 / 0104-7809.20164004399409$

\footnotetext{
*Universidade Federal do Rio de Janeiro (INJC/UFRJ). Rio de Janeiro, RJ. Brasil.

**Universidade Federal de Goiás. Goiânia, GO. Brasil.

As autoras declaram não haver conflitos de interesse.
} 


\section{INTRODUÇÃO}

A transição alimentar culminou em um consumo alimentar inadequado (pouco nutritivo e alto teor energético), por mais de $90,0 \%$ da população brasileira ${ }^{1}$, condição que indica a necessidade de adoção de favorecer comportamentos mais saudáveis ${ }^{2}$. Como consequência observa-se um aumento das prevalências de sobrepeso e obesidade, sobretudo em crianças e adolescentes, assim como retratado na Pesquisa Nacional de Saúde do Escolar em que se observou a prevalência de $23,0 \%$ de sobrepeso e de $7,3 \%$ de obesidade, com destaque às regiões Sul e Sudeste do país ${ }^{3}$.

Com vistas a reverter esse quadro e estimular a promoção da alimentação saudável no ambiente escolar torna-se essencial o desenvolvimento de ações de educação alimentar e nutricional - EAN - "prática contínua e permanente transdisciplinar e multiprofissional para se promover saúde e prevenir doenças, além de estimular mudanças no padrão alimentar e atividade física de forma voluntária e autônoma nesse e outros espaços ${ }^{4 \prime \prime}$.

A oferta da alimentação saudável e adequada com inserção da EAN no processo de ensino e aprendizagem no âmbito educacional público, incluindo ainda a realização do diagnóstico e acompanhamento do estado nutricional dos escolares, integra as ações do Programa Nacional de Alimentação Escolar (PNAE) ${ }^{5}$, o que indica resultados favoráveis na formação sobre práticas alimentares saudáveis com vistas à modificação do consumo alimentar e a redução do excesso de peso ${ }^{6}$. A escola representa um ambiente propício para o desenvolvimento destas ações, como cenário coparticipante da formulação de políticas públicas e tende a possibilitar a socialização e continuidade das informações sobre a tão necessária mudança de comportamento nesse contexto ${ }^{7,8}$.

A avaliação da prática alimentar de escolares é de grande relevância, entretanto existem poucos instrumentos validados no Brasil, para esse fim. Um deles, o Questionário Alimentar do Dia Anterior (QUADA), foi validado para crianças de sete a 10 anos, com vistas a obter informações do consumo atual de alimentos, sem dar ênfase à quantidade ou frequência de consumo e organiza as refeições em ordem cronológica, por meio de ilustrações que permitem maior contextualização da criança no tempo e espaço, para relatar sua prática alimentar do dia anterior ${ }^{9,10}$ e ser mais fidedigno.

Nesta perspectiva, como se prevê no PNAE, com vistas a alcançar seus princípios e diretrizes, e diante da escassez de estudos nessa área em Goiânia, Goiás, observou-se a indispensabilidade em investigar a possível associação entre intervenção nutricional educativa, alimentação e antropometria. O objetivo do presente trabalho foi avaliar a influência de um programa de intervenção nutricional educativa no hábito alimentar e indicadores antropométricos de alunos da primeira fase do ensino fundamental de uma escola pública federal.

\section{MÉTODOS}

\section{Tipo de estudo, população e amostra}

Estudo quantitativo prospectivo, com análise temporal de intervenção nutricional educativa, do tipo "antes" e "depois", em um período de cinco meses entre diagnóstico e realização da ação educativa. Foi realizado, entre os meses de abril a outubro de 2012, no Centro de Ensino e Pesquisa Aplicada à Educação (CEPAE), da Universidade Federal de Goiás (UFG), instituição pública que atende alunos dos ensinos fundamental e médio.

A população do estudo foi composta por 213 alunos da primeira fase do ensino fundamental ( $2^{\circ}$ ao $5^{\circ}$ ano). Atenderam aos critérios de inclusão: 1) autorização pelos pais, por meio da assinatura do Termo de Consentimento Livre e Esclarecido (TCLE) $(n=176) ; 2)$ participação de todas as ações propostas - três atividades educativas; duas avaliações antropométricas, inicial e final e duas aplicações do QUADA - $(n=92)$, constituindo-se a amostra final da pesquisa.

\section{Variáveis sociodemográficas e antropométricas}

Realizou-se caracterização sociodemográfica da família dos estudantes pesquisados utilizandose instrumento adaptado da Pesquisa Nacional 
de Saúde do Escolar (PeNSE) ${ }^{11}$ e da Associação Brasileira de Empresas de Pesquisa ${ }^{12}$. Obtevese as seguintes informações auto-referidas: data de nascimento, gênero, cor/raça, composição familiar, escolaridade dos pais e chefe da família, religião e posse de itens, por meio de entrevista telefônica, até a terceira tentativa, em dias e horários diferentes.

As variáveis antropométricas obtidas foram peso e altura, conforme técnica padronizada ${ }^{13}$. Calculou-se, por sua vez, o Índice de Massa Corporal (IMC) e estabeleceu-se o diagnóstico nutricional, com auxílio do software WHO AntroPlus versão 1.0.4 ${ }^{14}$.

\section{Hábito alimentar}

Utilizou-se o Questionário Alimentar do Dia Anterior (QUADA), previamente validado $^{9,10}$, aplicado como um estudo dirigido, por três auxiliares treinados, em cada série separadamente. Os alunos foram esclarecidos e enfatizou-se a importância de fornecerem informações verdadeiras e não interferirem nas respostas dos colegas ${ }^{10}$.

Os alimentos presentes no QUADA foram agrupados conforme recomendado no Guia Alimentar para a população brasileira, vigente na época do estudo ${ }^{15}$, com adaptações, em oito grupos: 1) iogurtes/leite e derivados/leite com achocolatado; 2) batata frita/ sanduíche/pizza/ salgado; 3) refrigerantes/ doces e guloseimas/ suco artificial; 4) feijão; 5) Arroz/ pão/ macarrão/ bolacha salgada (cereais); 6) ovos/ carnes bovina e de frango; 7) peixe e frutos do mar; 8) verduras/hortaliças e frutas.

A última parte do QUADA permite avaliar como o aluno "se sente" em relação a quatro grupos de alimentos (arroz/feijão; refrigerantes; frutas e verduras/hortaliças) ${ }^{10}$. Para realizar uma observação qualitativa, utilizou-se a classificação em alimentos de risco e proteção à saúde do Sistema de Vigilância Alimentar e Nutricional (SISVAN), do Ministério da Saúde ${ }^{16}$.

\section{Intervenção nutricional educativa}

As ações educativas foram pactuadas juntamente com a equipe de professores, coordenadores e gestão CEPAE, durante as reuniões da semana de planejamento pedagógico que antecipou a realização do estudo, fundamentadas nos materiais técnicos e legislação da área ${ }^{4,5,15}$.

A proposta teve como premissa básica a aplicação do modelo transteórico de acompanhamento dos estágios de mudança de comportamento, que inclui a identificação dos seus determinantes até a possibilidade de efetiva transformação das ações de EAN na modificação dos hábitos alimentares dos escolares ${ }^{17}$.

Reforça-se ainda que segundo $\operatorname{Boog}^{18}$, pesquisadora experiente da área, que atividades de EAN com fins de promoção da saúde devem ser compreendidas como práticas educativas, transformadoras da realidade social, centrada no indivíduo e nas coletividades.

Diante do exposto, e reconhecendo-se a efetividade de ações educativas em alimentação e nutrição na mudança de comportamento alimentar ${ }^{7,8}$, a intervenção proposta no presente trabalho foi dividida em seis etapas: 1) Pactuação das ações junto à gestão pedagógica; 2) Avaliação do estado nutricional; 3) Identificação do hábito alimentar dos estudantes; 4) Análise dos dados e planejamento da intervenção em alimentação e nutrição; 5) Implantação do programa de intervenção educativa com vistas à promoção da alimentação saudável e, 6) Avaliação do impacto do programa.

Ao término do desenvolvimento de todas as ações propostas, incluindo a primeira antropometria e o programa de ações educativas, com duração de cinco meses, aplicou-se o questionário QUADA novamente para identificar alterações no hábito alimentar e realizou-se uma nova avaliação antropométrica.

Para intervenção educativa foram propostos três grandes blocos, realizadas com os alunos por série, dentro e fora da sala de aula:

Bloco 1: Conhecendo o PNAE com o objetivo de apresentar o programa, sua importância e atribuições do nutricionista. Para as primeiras séries ( $2^{\circ}$ e $3^{\circ}$ anos) desenvolveu-se um teatro de fantoches e para os alunos maiores $\left(4^{\circ}\right.$ e $5^{\circ}$ anos) realizou-se roda de conversa. Ao final da atividade aplicou-se uma avaliação por turma para verificar o aprendizado.

Bloco 2: Mercado Saudável para apresentação dos grupos de alimentos propostos pelo Guia Alimentar ${ }^{15}$ e composição de uma 
alimentação saudável. Avaliou-se como evoluiu a compreensão da proposta das escolhas saudáveis, por meio das "compras" efetuadas antes e após a discussão do tema da ação.

Bloco 3: Gincana ativa e nutritiva com o objetivo de apresentar os grupos de alimentos, de acordo com sua função (energéticos, construtores e reguladores), por meio de circuitos de brincadeiras desportivas.

\section{Análise dos dados e aspectos éticos}

O banco de dados foi construído em Excel $^{\circledR}$, versão para Office 2007 e suas análises em

\section{RESULTADOS}

Do total de 92 alunos que compuseram a amostra, pouco mais que a maioria, era do gênero feminino $(55,44 \%, \mathrm{n}=51)$ (Tabela 1$)$.

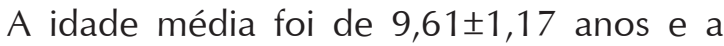
mediana de 10 anos, representando 33,70\% $(n=31)$ da amostra.

Para a caracterização sociodemográfica $61,96 \% \quad(n=57)$ da amostra participou da entrevistatelefônicaerespondeuaoquestionário. A maioria dos alunos foi classificada como: cor/ raça parda (57,90\%), religião católica (54,39\%), residia com pais $(68,42 \%)$ e possuía de um a dois irmãos (71,94\%). A classe social predominante foi a classe B $(54,38 \%)$, considerando-se o somatório das classes B1 e B2. No que se refere à escolaridade dos responsáveis, observouse que $49,12 \%$ das mães e $61,41 \%$ dos pais completaram até 12 anos de estudo (ensino médio), e destes, quase $10,0 \%$ e $20,00 \%$, das mães e pais, respectivamente, com até 8 anos.

Ao avaliar o estado nutricional por IMC observou-se que a maioria foi classificada como eutrófica, com percentual importante de excesso de peso (sobrepeso e obesidade), antes e após à intervenção nutricional (Tabela 2).

Os dados antropométricos apresentaram uma evolução ascendente, ao avaliar antes e após à educação nutricional, em que o peso mínimo variou de 18 a 19,4Kg e o máximo de 70,3 a $81,1 \mathrm{Kg}$; a altura mínima variou de 1,16 a $1,21 \mathrm{~m}$ e a máxima de 1,56 a 1,65m. Observou-
Epi Info, versão 3.4.2, incluindo a análise de consistência. Realizou-se análise descritiva para verificar associação entre hábito alimentar e parâmetros antropométricos antes e após a aplicação do programa de intervenção nutricional educativa empregaram-se o teste qui-quadrado, teste exato de Fisher e o KruskallWallis, considerando o nível de significância de $\mathrm{p}<0,05$. O projeto foi aprovado pelo Comitê de Ética em Pesquisa da Universidade Federal de Goiás, sob protocolo no 019/2012. Todos os participantes foram autorizados a participar após assinatura do Termo de Consentimento Livre e Esclarecido pelos responsáveis.

se também que o IMC mínimo variou de 12,71 a $12,42 \mathrm{Kg} / \mathrm{m}^{2}$ e o máximo variou de 29,64 a $31,29 \mathrm{Kg} / \mathrm{m}^{2}$. As medidas antropométricas foram distribuídas em intervalos interquartis antes e após intervenção educativa e observouse um aumento da média de peso e do IMC, entretanto sem significância estatística, e um ganho em estatura significativo $(p<0,0001)$ (Tabela 3).

Em relação às modificações nos hábitos alimentares após as ações educativas, observouse diminuição significativa do consumo dos alimentos dos grupos 1 (iogurte, leite e derivados) e 8 (frutas, verduras e hortaliças), no café da manhã e aumento significativo do grupo 3 (refrigerantes, doces, guloseimas e suco artificial). No lanche da manhã (colação) observou-se aumento significativo no consumo dos alimentos dos grupos 3 e 6 (ovos, carne bovina e frango) e redução significativa do grupo 5 (arroz, pão, macarrão e bolacha salgada) (Tabela 4).

Ao avaliar o almoço observou-se como aspecto positivo o aumento significativo no consumo dos alimentos dos grupos 5 e 8 e a manutenção do consumo daqueles que compõem o grupo 3. Como aspecto negativo, observou-se redução significativa no consumo dos grupos 4 (feijão) e 7 (peixe/derivados) e aumento significativo do grupo 2 (batata frita, pizza, sanduíche e salgado) (Tabela 4).

Ao avaliar o lanche da tarde, constatou-se o 
aumento significativo do consumo de iogurte, leite e derivados e frutas, verduras e hortaliças, em contrapartida, reduziu o consumo de arroz, pão, macarrão e bolacha salgada. Em relação ao jantar notou-se variação positiva com aumento significativo do consumo dos alimentos dos grupos 1, 4 e 6. Entretanto, apresentou redução negativa significativa do consumo de alimentos do grupo 8 (frutas, verduras e hortaliças) (Tabela 4). Ao comparar o grau de satisfação antes e após a intervenção, destaca-se o aumento significativo da satisfação em relação às frutas e redução significativa em relação aos refrigerantes, verduras, arroz e feijão (Tabela 5).

Tabela 1 - Estratificação da amostra, segundo gênero, por série da primeira fase do ensino fundamental. Centro de Pesquisa e Ensino Aplicado à Educação, Universidade Federal de Goiás (CEPAE/UFG). Goiânia, 2012.

\begin{tabular}{lcccccc}
\hline \multicolumn{1}{c}{ Série } & \multicolumn{2}{c}{ Masculino } & \multicolumn{2}{c}{ Feminino } & \multicolumn{2}{c}{ Total } \\
& $\mathbf{n}$ & \% & $\mathbf{n}$ & \% & $\mathbf{n}$ & \% \\
\hline $\mathbf{2}^{\mathbf{0}}$ Ano & 08 & 42,11 & 11 & 57,89 & 19 & 100,00 \\
$\mathbf{3}^{\mathbf{0}}$ Ano & 13 & 54,17 & 11 & 45,83 & 24 & 100,00 \\
$\mathbf{4}^{\mathbf{o}}$ Ano & 14 & 42,42 & 19 & 57,58 & 33 & 100,00 \\
$\mathbf{5}^{\mathbf{0}}$ Ano & 06 & 37,50 & 10 & 62,50 & 16 & 100,00 \\
\hline
\end{tabular}

Tabela 2 - Estado Nutricional de escolares da primeira fase do ensino fundamental, antes e após intervenção nutricional educativa. Centro de Pesquisa e Ensino Aplicado à Educação, Universidade Federal de Goiás (CEPAE/UFG). Goiânia, 2012.

\begin{tabular}{llcccc}
\hline \multicolumn{1}{c}{ Classificação do estado nutricional } & \multicolumn{2}{c}{ Antes } & \multicolumn{2}{c}{ Após } \\
& $\mathbf{n}$ & \% & $\mathbf{n}$ & $\%$ \\
\hline Magreza & 11 & 11,96 & 14 & 15,22 \\
Eutrofia & 63 & 68,48 & 58 & 63,04 \\
Sobrepeso & 13 & 14,13 & 13 & 14,13 \\
Obesidade & 05 & 05,42 & 07 & 07,61 \\
\hline
\end{tabular}

n: número; \%: percentual

Tabela 3 - Variáveis antropométricas de escolares da primeira fase do ensino fundamental, antes e após intervenção educativa. Centro de Pesquisa e Ensino Aplicado à Educação, Universidade Federal de Goiás (CEPAE/UFG). Goiânia, 2012.

\begin{tabular}{|c|c|c|c|c|c|c|c|c|c|}
\hline \multirow{2}{*}{ Variáveis } & \multicolumn{4}{|c|}{ Antes - Intervalo Interquartil } & \multicolumn{5}{|c|}{ Depois - Intervalo Interquartil } \\
\hline & $\begin{array}{l}\text { Média } \\
\pm \text { DPa }\end{array}$ & $25^{\circ}$ & $75^{\circ}$ & $\mathbf{X}^{\mathbf{b}}$ & $\begin{array}{l}\text { Média } \\
\pm \text { DPa }\end{array}$ & $25^{\circ}$ & $75^{\circ}$ & $\mathbf{X}^{\mathbf{b}}$ & $\mathbf{p}^{\mathrm{c}}$ \\
\hline Peso (kg) & $\begin{array}{l}30,79 \\
\pm 8,24\end{array}$ & 24,60 & 36,25 & 28,20 & $\begin{array}{l}34,98 \\
\pm 10,00\end{array}$ & 27,95 & 41,70 & 32,90 & 0,16 \\
\hline Altura (m) & $\begin{array}{l}1,34 \\
\pm 0,09\end{array}$ & 1,28 & 1,40 & 1,33 & $\begin{array}{l}1,41 \\
\pm 0,10\end{array}$ & 1,34 & 1,46 & 1,39 & 0,0000 \\
\hline $\mathrm{IMC}^{\mathrm{d}}\left(\mathrm{Kg} / \mathrm{m}^{2}\right)$ & $\begin{array}{r}16,90 \\
\pm 2,96\end{array}$ & 14,81 & 17,68 & 16,06 & $\begin{array}{r}17,33 \\
\pm 3,28\end{array}$ & 15,08 & 18,64 & 16,76 & 0,41 \\
\hline
\end{tabular}

${ }^{a}$ Desvio Padrão; ${ }^{b} X=$ Mediana; ${ }^{\text {TTeste kruskall-Wallis; }{ }^{d} I M C-I ́ n d i c e ~ d e ~ M a s s a ~ C o r p o r a l . ~}$ 
Tabela 4 - Consumo da alimentação escolar de alunos da primeira fase do ensino fundamental, antes e após intervenção educativa. Centro de Pesquisa e Ensino Aplicado à Educação, Universidade Federal de Goiás (CEPAE/UFG). Goiânia, 2012.

\begin{tabular}{|c|c|c|c|c|c|c|}
\hline \multirow{2}{*}{ Refeição } & \multirow{2}{*}{ Grupo alimentar ${ }^{1}$} & \multicolumn{2}{|c|}{ Antes } & \multicolumn{2}{|c|}{ Após } & \multirow[t]{2}{*}{ p } \\
\hline & & $\mathrm{n}$ & $\%$ & $\mathrm{n}$ & $\%$ & \\
\hline \multirow[t]{6}{*}{ Café da manhã } & G1 & 72 & 78,26 & 54 & 58,69 & $0,001^{2}$ \\
\hline & G2 & 01 & 1,09 & 05 & 5,44 & $0,054^{3}$ \\
\hline & G3 & 14 & 15,21 & 22 & 23,91 & $0,002^{2}$ \\
\hline & G5 & 32 & 34,78 & 40 & 43,47 & $0,173^{2}$ \\
\hline & G6 & 03 & 3,26 & 06 & 6,52 & $0,185^{3}$ \\
\hline & G8 & 15 & 16,30 & 09 & 9,78 & $0,036^{3}$ \\
\hline \multirow[t]{6}{*}{ Lanche da manhã } & G1 & 05 & 5,44 & 03 & 3,26 & $0,84^{3}$ \\
\hline & G2 & 04 & 4,34 & 04 & 4,34 & $0,83^{3}$ \\
\hline & G3 & 22 & 23,91 & 53 & 57,60 & $0,007^{3}$ \\
\hline & G4 & 70 & 76,08 & 59 & 64,13 & $0,036^{3}$ \\
\hline & G5 & 09 & 9,78 & 49 & 53,26 & $0,024^{3}$ \\
\hline & G6 & 23 & 25,00 & 01 & 1,09 & $0,75^{3}$ \\
\hline \multirow[t]{8}{*}{ Almoço } & G1 & 03 & 8,26 & 04 & 4,34 & $0,87^{3}$ \\
\hline & G2 & 09 & 9,78 & 10 & 10,86 & $0,007^{3}$ \\
\hline & G3 & 52 & 56,52 & 52 & 56,52 & $0,017^{3}$ \\
\hline & G4 & 69 & 75,00 & 62 & 67,39 & $<0,001^{3}$ \\
\hline & G5 & 80 & 86,95 & 81 & 88,04 & $<0,001^{3}$ \\
\hline & G6 & 73 & 79,34 & 69 & 75,00 & $0,181^{2}$ \\
\hline & G7 & 06 & 6,52 & 03 & 3,26 & $0,010^{3}$ \\
\hline & G8 & 43 & 46,73 & 47 & 51,09 & $0,035^{2}$ \\
\hline \multirow[t]{7}{*}{ Lanche da tarde } & G1 & 34 & 36,95 & 42 & 45,65 & $0,042^{3}$ \\
\hline & G2 & 13 & 14,13 & 13 & 14,13 & $0,083^{3}$ \\
\hline & G3 & 49 & 53,26 & 46 & 50,00 & $0,296^{2}$ \\
\hline & G4 & 01 & 1,09 & 01 & 1,09 & $0,989^{3}$ \\
\hline & G5 & 37 & 40,21 & 32 & 34,78 & $0,006^{2}$ \\
\hline & G6 & 07 & 7,60 & 06 & 6,52 & $0,387^{3}$ \\
\hline & G8 & 22 & 23,91 & 25 & 27,17 & $0,027^{2}$ \\
\hline
\end{tabular}




\begin{tabular}{ccccccc}
\hline Jantar & G1 & 05 & 5,44 & 07 & 7,60 & $0,002^{3}$ \\
\hline G2 & 19 & 20,65 & 16 & 17,39 & $0,067^{2}$ \\
G3 & 34 & 36,95 & 38 & 41,30 & $0,195^{2}$ \\
G4 & 39 & 42,39 & 45 & 48,91 & $<0,001^{2}$ \\
G5 & 59 & 64,13 & 66 & 71,73 & $0,076^{2}$ \\
G6 & 46 & 50,00 & 55 & 81,52 & $0,001^{2}$ \\
G7 & 05 & 5,44 & 04 & 4,34 & $0,797^{3}$ \\
G8 & 28 & 30,43 & 27 & 29,34 & $0,017^{2}$ \\
\hline
\end{tabular}

${ }^{1} \mathrm{G} 1=$ logurte, leite e derivados e leite com chocolate; G2 = batata frita, pizza, sanduíche e salgado; G3= refrigerante, doce, guloseimas e suco artificial; G4= feijão; G5= arroz, pão, macarrão e bolacha salgada; G6= ovos, carne bovina e frango; G7= peixe; G8= frutas, verduras e hortaliças.

${ }^{2}$ Teste Qui-quadrado; ${ }^{3}$ Teste Exato de Fisher.

Tabela 5 - Perspectiva "como se sente" de escolares da primeira fase do ensino fundamental sobre alguns grupos de alimentos, antes e após intervenção educativa. Centro de Pesquisa e Ensino Aplicado à Educação, Universidade Federal de Goiás (CEPAE/UFG). Goiânia, 2012.

\begin{tabular}{lcrrrr}
\hline \multirow{2}{*}{ Grupos de alimentos } & \multicolumn{2}{c}{ Antes } & \multicolumn{2}{c}{ Após } & \multicolumn{1}{c}{$\mathbf{p}^{*}$} \\
& $\mathbf{n}$ & $\mathbf{\%}$ & $\mathbf{n}$ & $\mathbf{0}$ & \\
\hline Arroz e feijão & 80 & 86,95 & 76 & 82,61 & 0,014 \\
Refrigerantes & 72 & 78,26 & 67 & 72,82 & 0,005 \\
Frutas & 81 & 88,04 & 84 & 91,30 & $<0,001$ \\
Verduras/Hortaliças & 57 & 61,95 & 53 & 57,60 & 0,001 \\
\hline
\end{tabular}

${ }^{*}$ Teste de Qui-quadrado.

\section{DISCUSSÃO}

O presente estudo foi conduzido com um grupo de estudantes com mediana de idade de 10 anos, maioria do sexo feminino, parda, católica, que moravam com o pai, mãe e até dois irmãos, da classe econômica B, pais com até 12 anos de estudo e classificação do estado nutricional por antropometria de eutrofia, apesar um quinto apresentar excesso de peso.

Ao identificar o hábito/prática alimentar constatou-se que os escolares apresentaram consumo aumentado de alimentos do grupo dos refrigerantes, doces, guloseimas, suco artificial e da batata frita, pizza, sanduíche e salgado, além do baixo consumo de frutas, verduras e hortaliças, apesar da adesão ao PNAE.

Verificou-se que as ações educativas executadas possibilitaram mudanças no conhecimento sobre nutrição entre os escolares e permitiu modificações positivas e negativas em sua alimentação, com aumento e/ou redução do consumo de alimentos considerados fatores de risco e de proteção, dependendo da refeição avaliada.

Ao avaliar a influência do programa de intervenção nutricional educativa sobre os indicadores antropométricos verificou-se 
que houve aumento da média de peso e do IMC sem significância estatística e um ganho estatural significativo.

A escolaridade dos pais, em especial, a materna e a classe social são fatores socioeconômicos importantes na determinação da saúde do escolar, sendo observado, por exemplo, que mães com escolaridade menor aumentam a probabilidade de crianças não consumirem uma alimentação de qualidade tanto pelo acesso aos alimentos quanto ao conhecimento para as escolhas mais saudáveis ${ }^{19}$.

A qualidade inadequada da alimentação de crianças sofre influência de vários fatores, incluindo a menor escolaridade do cuidador e/ ou responsável, o hábito de realizar as refeições em frente à televisão, falta de atividade física e a própria avaliação negativa dos responsáveis sobre a alimentação das crianças. As atitudes dos pais em relação à alimentação dos filhos são importantes para fomentar práticas saudáveis de alimentação ${ }^{20}$.

Reconhece-se que a formação dos hábitos alimentares se inicia nos primeiros anos de vida e apesar da escola exercer papel primordial nessa fase, seu estabelecimento se dá essencialmente no seio familiar, por meio das aprendizagens e experiências vivenciadas nesse meio ${ }^{21}$. Apesar de não ter sido objeto direto de estudo do presente trabalho correlacionar a escolaridade materna e/ou cuidador e sua influência no comportamento alimentar sugere-se que tal aspecto pode ter condicionado os achados deste.

Estudos no Brasil indicam uma alta prevalência do excesso de peso entre crianças $^{6,12}$. A prevalência de excesso de peso (sobrepeso e obesidade) encontrada no presente estudo, antes e após a intervenção educativa foi de $19,55 \%$ e $21,74 \%$, respectivamente, e corrobora com achados de pesquisa semelhante realizada em Florianópolis, SC, em que antes da intervenção educativa a prevalência de excesso de peso foi de $21,8 \%$ e depois de $23,6 \%{ }^{23}$.

Entretanto, em estudo que realizou avaliação antropométrica em escolares submetidos a um programa de educação nutricional por um período de 10 meses, em um escola pública, observou que após intervenção nutricional e de atividade física, comparando-se a uma escola controle, diferença significativa no aumento da massa gorda no grupo controle em relação ao grupo de intervenção ${ }^{6}$. Isso evidencia a discrepância entre a abordagem meramente educativa do ponto de vista nutricional e a abordagem associada à atividade física. Atividades de EAN isoladamente não produzem efeitos positivos significativos na redução do IMC ou composição corporal entre escolares, somente quando associados à prática de atividade física ${ }^{24}$.

No que se refere ao resultado encontrado de evolução estatural ascendente, estudo realizado em escolares de Pernambuco ${ }^{5}$ revela uma redução do déficit de estatura, apresentandose em concordância ao presente trabalho. Segundo os autores, esta condição reflete a melhoria das condições sociais e econômicas proporcionadas por políticas públicas ${ }^{25}$.

Sabe-se, ainda, que uma intervenção isolada em uma única área não é suficiente para resolver o problema de excesso de peso em uma comunidade escolar. Além disso, deve-se considerar que o tempo entre a primeira e a segunda avaliação antropométrica (cinco meses), pode não ter sido suficiente para uma mudança significativa dos índices antropométricos, bem como o número de atividades desenvolvidas em cada grupamento, apenas três. Desta forma, destaca-se que a avaliação antropométrica pode não ser um método apropriado para avaliar intervenções educativas desenvolvidas em curto espaço de tempo ${ }^{7}$. O pouco tempo de intervenção pode ser uma limitação quando se quer avaliar alguma mudança de comportamento e/ou antropométrica, em especial.

Apesar da existência de diferentes teorias de aprendizagens com enfoque em comportamentos de saúde, sabe-se que a teoria cognitivo-comportamental é uma estratégia bem empregada com resultados favoráveis para mudança de conduta nessa área ${ }^{26}$, em especial, de hábito alimentar, em que o reforço do comportamento saudável, incluindo até mesmo no preparo da sua própria refeição, aumenta a confiança da pessoa no processo de suas escolhas, com ênfase na preexistência de determinada enfermidade e reforça a credibilidade de quem transmite a mensagem educativa $^{27}$.

Como fruto do trabalho educativo no 
presente estudo, observou-se o aumento do consumo de frutas, verduras e legumes no almoço e lanche da tarde. Entretanto esse percentual foi considerado baixo, uma vez que esse grupo alimentar deveria estar diariamente presente no cotidiano de todas as crianças, segundo recomendações ${ }^{16}$.

No sul do país coletaram-se dados de escolares, matriculados em escolas públicas e particulares do ensino fundamental com idade entre seis e 10 anos, em municípios de Santa Catarina a respeito do consumo de frutas e hortaliças e seus resultados confirmam os achados do presente estudo ${ }^{22}$. Tendo como parâmetro as recomendações do referido Guia $^{16}$, apenas 2,7\% dos escolares consumiram cinco ou mais vezes frutas e hortaliças/dia. Além disso, 26,6\% não consumiam esses alimentos nenhuma vez por dia, 48,1\% e 46,3\% omitiram, respectivamente, frutas e hortaliças ${ }^{28}$.

Outroaspectoquemereceudestaquefoioalto percentual de consumo de refrigerante, doces, guloseimas e suco artificial, principalmente, no lanche da manhã $(57,60 \%)$, com aumento do consumo após a intervenção nutricional. Em São Luís do Maranhão, pesquisa realizada em escolas públicas e privadas, com 570 alunos de idade entre nove e 16 anos, também verificou um consumo alimentar elevado de açúcares e doces $(69,40 \%)$. Além disso, a ingestão de refrigerantes e sucos industrializados foi maior do que sucos naturais ${ }^{29}$. Os resultados da POF 2008-20091 também indicaram alto consumo de doces, refrigerantes, pizza, salgados fritos e assados e foram maiores nas categorias de rendas mais altas.

$\mathrm{Na}$ avaliação do hábito alimentar notou-se que a técnica proposta para a aplicação do QUADA permitiu e facilitou a recuperação da memória dos acontecimentos junto às crianças, no que se diz respeito às refeições realizadas. O curto espaço de tempo entre a aplicação do instrumento e a realização das refeições contribuiu com a melhoria da retenção da memória ${ }^{10}$.

Durante a aplicação do QUADA 9,10 pode ter ocorrido a subnotificação de determinados alimentos pela inexistência da sua figura no questionário, como àqueles típicos da região goiana: pamonha, caldos, galinhada, empadão, pão de queijo. Nestes casos, os alunos que questionavam onde deveriam assinalar, foram orientados a marcar, separadamente, os ingredientes da preparação ou os alimentos que mais se aproximariam, do ponto de vista de macro e micronutrientes.

Observa-se, pois, que a imagem é fundamental para estimular a recuperação da memória, minimizando erros de recuperação desta. Portanto, não se sabe se a resposta fornecida pelas crianças estava contemplada ou não no questionário. Prevendo esse tipo de viés, estudiosos preocuparam-se com a problemática da subnotificação e elaboraram um Questionário de Frequência Alimentar Quantitativo (QFAQ), para crianças de sete a 10 anos, com criação de espaços para escreverem o alimento caso não estivesse na lista ${ }^{30}$.

O QUADA é um instrumento que avalia a ingestão atual do consumo alimentar, e este resultado é utilizado para estimar a média de consumo de um grupo. Entretanto, a alimentação de um indivíduo varia de acordo com seu cotidiano, sazonalidade, interesses e necessidades. Sendo assim, este questionário alimentar pode não avaliar e caracterizar com veracidade o consumo do público-alvo, uma vez que precisaria de outros dias alimentares para melhor representatividade ${ }^{9,10}$.

Em relação ao lanche da manhã, por se tratar de uma escola pública, que recebe recursos do Fundo Nacional de Desenvolvimento da Educação (FNDE) para execução do PNAE, esperava-se aumento do consumo de arroz, macarrão, bolacha salgada que são frequentemente oferecidos na alimentação escolar, como demonstrado em estudo sobre a temática, realizado em Minas Gerais ${ }^{31}$. Os autores observaram que $83,5 \%$ dos alunos consumiam, no ambiente escolar, alimentos que não eram provenientes do PNAE. Acreditase que o aumento do consumo de refrigerante, doces, guloseimas e suco artificial esteja relacionado à compra destes alimentos que são comercializados na porta da escola ${ }^{31}$.

Ações de educação alimentar e nutricional desenvolvidas mesmo que em um tempo relativamente curto de intervenção (cinco meses), podem apresentar alterações positivas na qualidade do consumo dos alunos. Todavia, intervenções em longo prazo, podem demonstrar ainda maior significância quanto 
à melhoria da qualidade, dos alimentos consumidos e de vida das crianças. Algumas das estratégias sugeridas pelos autores são: integração de ações educativas em nutrição no currículo escolar; incentivo à prática da atividade física; participação efetiva da comunidade escolar e da família, uma vez que estas interferem diretamente na formação dos hábitos de vida na infância ${ }^{23}$.

A Educação Alimentar e Nutricional $^{4}$ pode mostrar-se, fortemente, significativa na formação de hábitos alimentares saudáveis no âmbito escolar, uma vez que as crianças passam grande parte do dia na escola, como se observou em estudo realizado em Juiz de Fora em que avaliou conhecimento nutricional e comportamento alimentar após educação alimentar e nutricional em adolescentes e verificou melhoras significativas nos estágios de mudanças de ambos ${ }^{32}$.

Entretanto, as intervenções devem ter um sentido mais amplo, considerando ações integradas à mídia, às indústrias, e ao próprio sistema de saúde brasileiro com vistas à promoção da saúde ${ }^{6}$, que contribuem, por meio do uso de metodologias inovadoras de educação em saúde, com a investigação científica neste campo, ainda incipiente ${ }^{7}$.

\section{CONCLUSÃO}

Os escolares apresentaram uma prevalência elevada de excesso de peso e consumo aumentado de alimentos do grupo dos refrigerantes, doces, guloseimas, suco artificial e da batata frita, pizza, sanduíche e salgados, além do baixo consumo de frutas, verduras e hortaliças, mesmo após a intervenção nutricional. Em relação ao hábito/prática alimentar constatou-se que o programa de intervenção educativa favoreceu modificações positivas e negativas na alimentação dos escolares, com aumento e/ou redução do consumo de alimentos considerados fatores de risco e de proteção, dependendo da refeição avaliada. Entretanto, o desenvolvimento do programa educativo possibilitou mudanças no conhecimento sobre nutrição entre os escolares.

Entende-se que ações de Educação Alimentar e Nutricional devem ser contínuas e inseridas no planejamento pedagógico das disciplinas ministradas para escolares desta faixa etária, com vistas a possibilitar maior conhecimento e apropriação das informações sobre promoção da alimentação saudável na escola, além de melhores condições de ensino aprendizagem, culminando com a incorporação dos hábitos saudáveis no cotidiano de cada aluno.

\section{REFERÊNCIAS}

\footnotetext{
1. IBGE. Instituto Brasileiro de Geografia e Estatística. Pesquisa de Orçamentos Familiares (POF)- 2008-2009: análise do consumo alimentar pessoal no Brasil. Rio de Janeiro: IBGE; 2011. 150p. Disponível em: http://biblioteca.ibge.gov.br/visualizacao/livros/liv50063. pdf

2. Vargas ICS, Sichieri R, Sandre-Pereira G, Veiga GV. Avaliação de programa de prevenção de obesidade em adolescentes de escolas públicas. Rev Saúde Pública. 2011;45(1):59-68. DOI: http://dx.doi.org/10.1590/S0034-89102011000100007.

3. Araúo C, Toral N, Silva ACF, Velásquez-Melendez G, Dias AJR. Estado nutricional dos adolescentes e sua relação com variáveis sociodemográficas: Pesquisa Nacional de Saúde do Escolar (PeNSE), 2009. Ciênc Saúde Coletiva. 2010; 15(Supl.2): 3077-84. DOI: http://dx.doi.org/10.1590/S1413-81232010000800012

4. Brasil. Ministério do Desenvolvimento Social e Combate à Fome. Marco de referência de educação alimentar e nutricional para as políticas públicas. Brasilia, DF: MDS; Secretaria Nacional de Segurança Alimentar e Nutricional, 2012.

5. Brasil. Fundo Nacional de Desenvolvimento da Educação. Ministério da Educação. Resolução 26, de 17 de junho de 2013. Dispõe sobre o atendimento da alimentação escolar aos alunos da educação básica no âmbito do Programa Nacional de Alimentação Escolar - PNAE. Brasilia, DF: MEC, 2013.

6. Feferbaum R, Leone C, Nogueira RC, Cavalcanti PN, Cardoso EB, Serra MA. Avaliação antropométrica por bioimpedância de um programa de educação nutricional para escolares na faixa etária de 7-14 anos durante o período de 10 meses. Rev Bras Crescimento Desenvolv Hum. 2012; 22(3): 283-290. Disponível em: http://pepsic.bvsalud.org/pdf/rbcdh/v22n3/pt_02.pdf.

7. Ramos FP, Santos LAS, Reis, ABC. Educação alimentar e nutricional em escolares: uma revisão de literatura. Cad Saúde Pública. 2013;29(11):2147-61. DOI: http://dx.doi.org/10.1590/0102-311x00170112.
} 
8. Santos LAS. O fazer educação alimentar e nutricional: algumas contribuições para reflexão. Ciênc Saúde Coletiva. 2012;17(2): 45362.

9. Assis MAA, Kupek E, Guimarães D, Calvo MCM, Andrade DF, Bellisle F. Test-retest reliability and external validity of the Previous Day Food Questionnaire for 7- to 10-year-old school children. Appetite. 2008;51(1):187-93. DOI: 10.1016/j.appet.2008.02.014.

10. Assis MAA, Benedet J, Kerpel R, Vasconcelos FAG, Di Pietro PF, Kupek E. Validação da terceira versão do Questionário Alimentar do Dia Anterior (QUADA-3) para escolares de 6 a 11 anos. Cad Saúde Pública. 2009;25(8):1816-26. DOI: http://dx.doi.org/10.1590/ S0102-311X2009000800018.

11. Instituto Brasileiro de Geografia e Estatística. Pesquisa Nacional de Saúde do Escolar 2012. Rio de Janeiro: 2013 [acesso 20 Mar 2015]. Disponível em: http://www.ibge.gov.br/home/estatistica/populacao/pense/2012/

12. ABEP. Associação Brasileira de Empresas de Pesquisa. Critério de Classificação Econômica Brasil. São Paulo: ABEP, 2008 [acesso 26 jan 2012], Disponível em: http://www.abep.org/criterioBrasil.aspx.

13. Lohman TG, Roche AF, Martorell R. Antropometric standardization reference manual. Champaing, IL: Human kinetics books. 1988. $89 \mathrm{p}$.

14. WHO. WHO AnthroPlus for personal computers Manual: Software for assessing growth of the world's children and adolescents. Geneva: WHO, 2009. [acesso 10 mar 2015]. Disponível em: http://www.who.int/growthref/tools/who_anthroplus_manual.pdf.

15. Brasil. Ministério da Saúde. Guia alimentar para a população brasileira: promovendo a alimentação saudável. Brasília: Ministério da Saúde; 2006.

16. Brasil. Ministério da Saúde. Protocolos do Sistema de Vigilância Alimentar e Nutricional - SISVAN na assistência à saúde. Brasilia: Ministério da Saúde, 2008.

17. Toral N, Slater B. Abordagem do modelo transteórico no comportamento alimentar. Ciênc. Saúde Coletiva. 2017; 12(6): 1641-50. 18. Boog MCF. (2013) Educação em Nutrição: Integrando experiências. 1. (268p.) Ed. Campinas: Komedi Editora. ISBN: 9788575826775. 19. Molina MCB, Lopéz PM, Faria CP, Cade NV, Zandonade E. Preditores socioeconômicos da qualidade da alimentação de crianças. Rev Saúde Pública. 2010;44(5):785-92. DOI: http://dx.doi.org/10.1590/S0034-89102010005000036.

20. Momm N, Höfelmann DA. Qualidade da dieta e fatores associados em crianças matriculadas em um escola municipal em Itajaí, Santa Catarina. Cad Saúde Colet. 2014;22(1): 32-9. DOI: http://dx.doi.org/10.1590/1414-462X201400010006.

21. Viana V, Franco T, Morais C. O estado ponderal e o comportamento alimentar de crianças e jovens: influência do peso e das atitudes de controlo da mãe. Psicol Saúde Doenças. 2011;12(2): 267-79.

22. Rivera JA, Cossio TG, Pedraza LS, Aburto TC, Sánchez TG, Martorell R. Childhood and adolescent overweight and obesity in Latin America: a systematic review. Lancet Diabetes Endocrinol. 2014;2(4):321-332. DOI: http://dx.doi.org/10.1016/S2213-8587(13)701736.

23. Fernandes OS, Berbardo CO, Campos RMMB, Vasconcelos FAG. Avaliação do efeito da educação nutricional na prevalência de sobrepeso/obesidade e no consumo alimentar de escolares de ensino fundamental. J Pediatria. 2009;85(4):315-21. DOI: http://dx.doi. org/10.1590/S0021-75572009000400008.

24. Friedrich RR, Schuch I, Wagner MB. Efeito de intervenções sobre o índice de massa corporal em escolares. Rev Saúde Pública. 2012 abr;46(3):551-60. DOI: http://dx.doi.org/10.1590/S0034-89102012005000036.

25. Leal VS, Lira PIC, Menezes RCE, Oliveira J S, Sequeira LAS, Andrade SLLS, Batista-Filho M. Fatores associados ao declínio do déficit estatural em crianças e adolescentes em Pernambuco. Rev Saúde Pública. 2012;46(2):234-41. DOI: http://dx.doi.org/10.1590/S003489102012005000015.

26. Dobson KS, Dozois DJA. (2006). Fundamentos históricos e filosóficos das terapias cognitivo-comportamentais. In: K. S. Dobson (Org.). Manual de terapias cognitivos comportamentais. 2. ed. (p. 17-43). Porto Alegre: Artmed.

27. Peyrot M, Rubin RR. Behavioral and psychosocial interventions in diabetes. Diabetes Care. 2007;30:2433-40. DOI:10.2337/dc071222 .

28. Costa LCF, Vasconcelos FAG, Corso ACT. Fatores associados ao consumo adequado de frutas e hortaliças em escolares de Santa Catarina, Brasil. Cad Saúde Pública. 2012;28(6):1133-42. DOI: http://dx.doi.org/10.1590/S0102-311X2012000600012.

29. Conceição SIO, Santos CJN, Silva AAM, Oliveira TC. Consumo alimentar de escolares das redes pública e privada de ensino em São Luís, Maranhão. Rev Nutr. 2010;23(6):993-1004. DOI: http://dx.doi.org/10.1590/S1415-52732010000600006.

30. Hinnig PF, Mariath AB, Freaza SRM, Gambardella AMD, Bergamaschi DP. Construção de questionário alimentar para crianças de 7 a 10 anos. Rev Bras Epidemiol. 2014 jun;17(2):479-494. DOI: http://dx.doi.org/10.1590/1809-4503201400020015ENG.

31. Silva CAM, Marques LA, Bonomo E, Bezerra OMPA, Correa MS, Passos LSF, Souza AA, Barros BF, Souza DMS, Reis JA, Andrade NM. O Programa Nacional de Alimentação Escolar sob a ótica dos alunos da rede estadual de ensino de Minas Gerais, Brasil. Ciênc Saúde Coletiva. 2013;18(4):963-69. DOI: http://dx.doi.org/10.1590/S1413-81232013000400008.

32. Assis MM, Penna LF, Neves CM, Mendes APCC, Oliveira RMS, Netto MP. Avaliação do conhecimento nutricional e comportamento alimentar após educação alimentar e nutricional em adolescentes de Juiz de Fora-MG. HU Revista. 2014; 40(3 e 4):135-43.

Recebido em junho de 2015. Aprovado em novembro de 2016. 